\title{
LETTER
}

\section{Microbiota in ICU, not only a gut problem}

\author{
L. Alagna ${ }^{1 *} \mathbb{D}$, A. Bandera ${ }^{1,2}$, A. Patruno ${ }^{3}$, A. Muscatello ${ }^{1}$, G. Citerio ${ }^{3,4}$ and A. Gori ${ }^{1,2}$
}

@ 2019 Springer-Verlag GmbH Germany, part of Springer Nature

Dear Editor,

The editorial by Ruppé et al. [1] related to the article by Freedberg et al. [2] about gut microbiota in ICU patients describing the association between Enterococcus dominance in the gut at ICU admission and death was very interesting. This paper provides a useful insight for future research on gut microbiota in critically ill patients. Dysbiosis can be a trigger to promote many nosocomial infections [3] because of lack of equilibrium among bacteria species.

Given this finding, we deem that, in ICU setting, studies should not be limited to gut microbiota, but it could be crucial to investigate even lung microbiota considering the potential application in mechanically ventilated patients.

The role of lung microbiota in ICU mechanically ventilated patients is still underexplored. In fact, its potential to predict and to prevent infections in a clinical condition at high risk of complications related to mechanical ventilation (MV) such as ventilator-associated pneumonia (VAP) is unknown. Few data are available on lung microbiota during MV and on pathogens which eventually colonize the endotracheal tube (ETT) and cause VAP.

ETT is an interface between patient and ventilator, which can modify the oral microbiota composition, and can be colonized by commensal oral or respiratory bacteria. These bacteria can translocate to the lower airways and cause a high increase in the likelihood of VAP insurgence.

As described on Table 1 (part A), some studies have analyzed the colonization of dental plaque performed by standard culture techniques showing a change in microbial composition of the oral cavity and an incorporation of at least one respiratory pathogen potentially responsible for VAP (Staphylococcus aureus and P. aeruginosa). Other studies have analyzed the microbiota of dental plaque or the ETT directly, confirming a microbial shift in colonization with a predominance of non-oral bacteria during a different stage of intubation. In addition, the detection of some bacteria (i.e. Pseudomonas or $S$. aureus) in ETT correlate with worse outcome (see table for references).

Few preliminary studies of lung microbiota during MV are available (Table 1, part B). The studies showed a reduction in number and abundance of species during MV. The correlation between modification in microbiota composition and VAP development is not conclusive. Lu et al. [4] described a difference in constituent ratio of some bacteria (Klebsiella sp., Acinetobacter sp., Streptococcus $\mathrm{sp}$.) among patients with VAP compared with no VAP patients. Moreover, Zakharkina et al. [5] observed that patients with VAP have more profound dysbiosis

\footnotetext{
*Correspondence: laura.alagna@policlinico.mi.it

${ }^{1}$ Infectious Diseases Unit, Departement of Internal Medicine, Fondazione IRCCS Ca' Granda Ospedale Maggiore Policlinico, Milan, Italy

Full author information is available at the end of the article
}

\section{实 Springer}




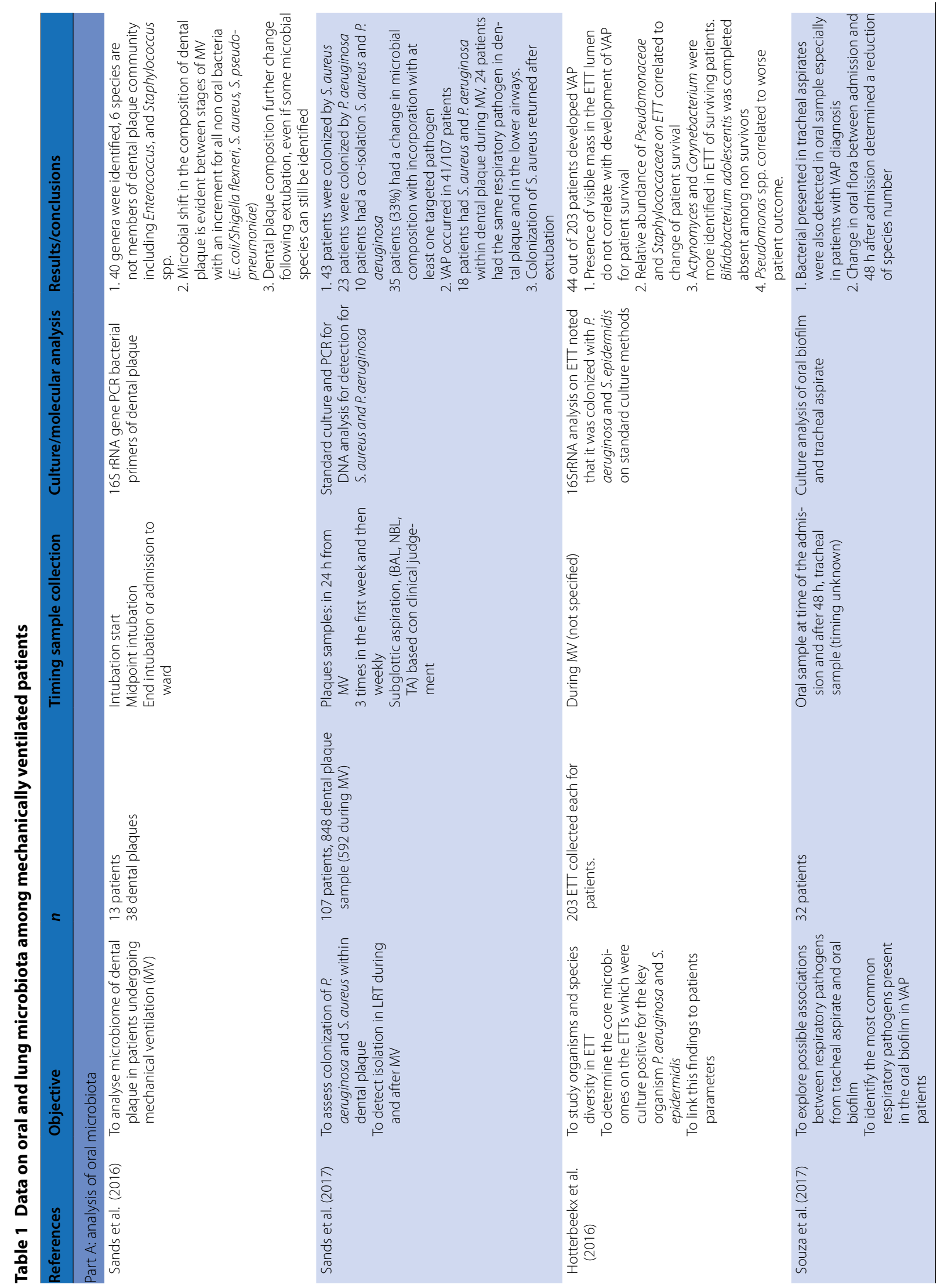




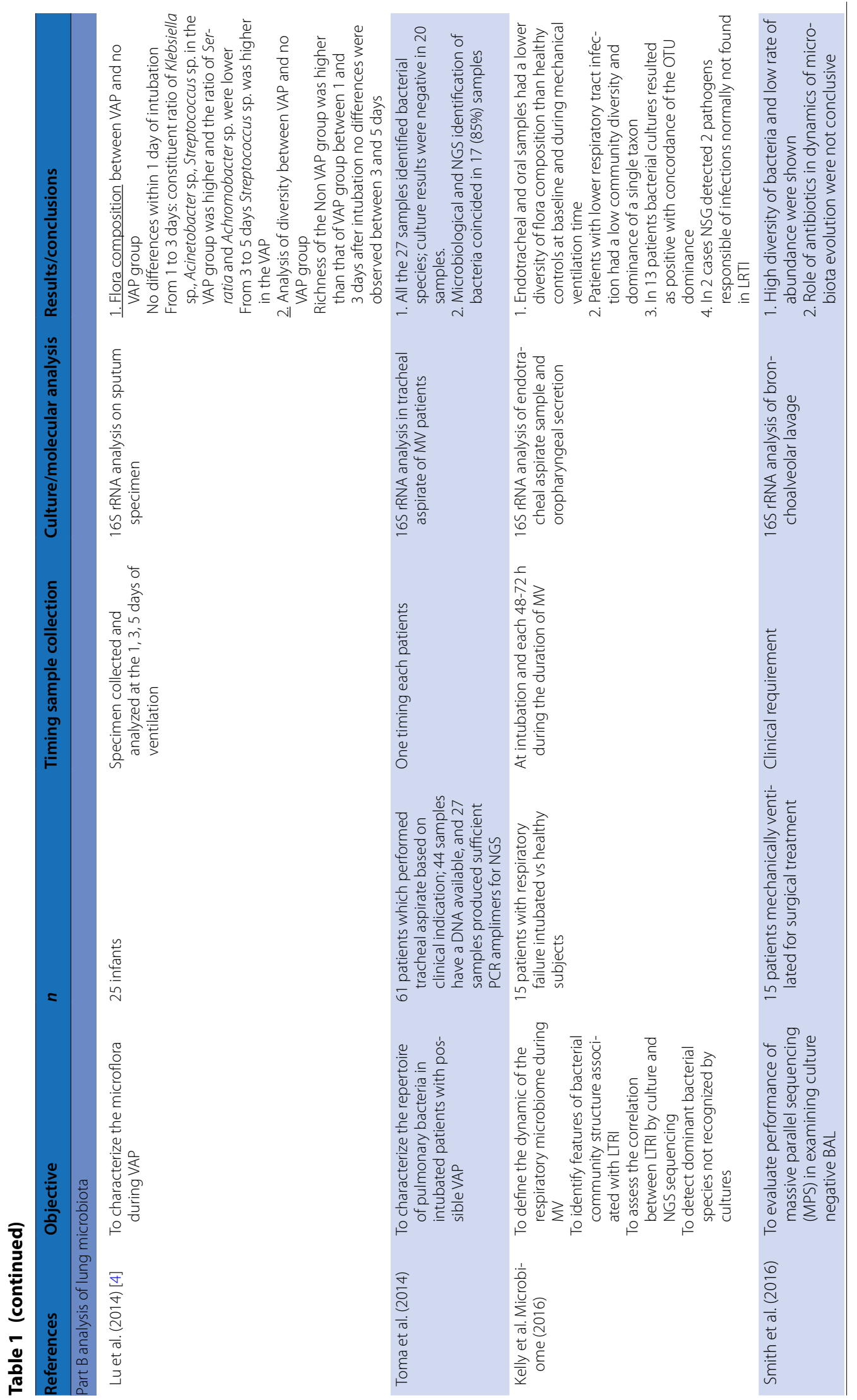




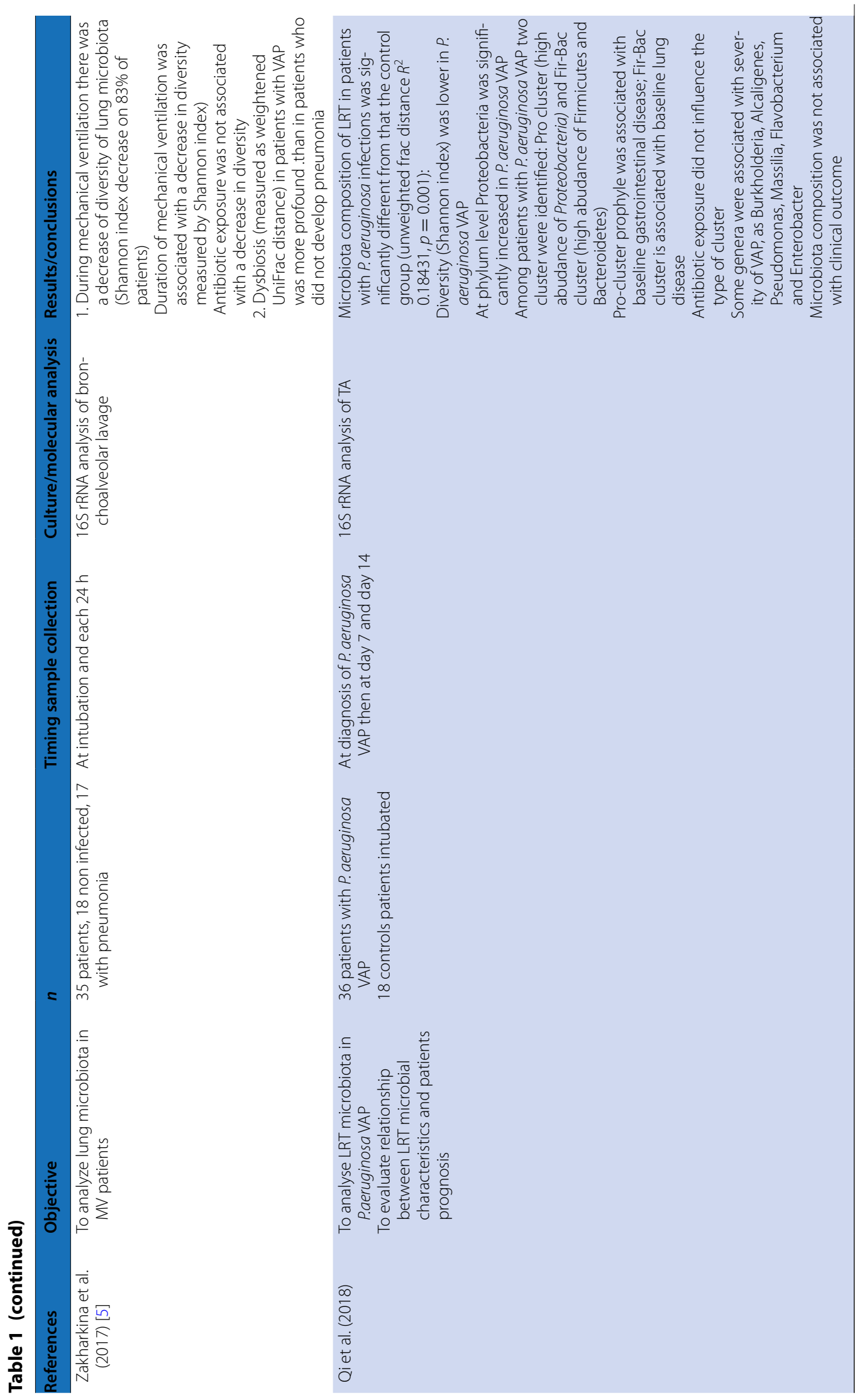


that controls patients. Exposure of antibiotics is not associated with changes in microbiota composition, but these data need to be confirmed,

This conclusion opens a large field of investigation addressed to understand how lung microbiota characteristic can predispose and correlate to VAP insurgence.

Further studies aimed at analyzing the change of lung microbiota during $\mathrm{MV}$ and to evaluate characteristics associated with VAP development are needed.

We think that the identification of basal risk factors associated with a higher likelihood ratio to develop VAP could be useful to implement measures of prevention and diagnosis. Studies on microbiota in ICU will be a challenge to improve patient management.

Publisher's Note Springer Nature remains neutral with regard to jurisdictional claims in published maps and institutional affiliations.

\begin{abstract}
Author details
${ }^{1}$ Infectious Diseases Unit, Departement of Internal Medicine, Fondazione IRCCS Ca' Granda Ospedale Maggiore Policlinico, Milan, Italy. ${ }^{2}$ Department of Pathophysiology and Transplantation, School of Medicine and Surgery, University of Milan, Milan, Italy. ${ }^{3}$ Neuro Intensive Care Unit, Department of Emergency and Intensive Care, San Gerardo Hospital, Monza, Italy. ${ }^{4}$ School of Medicine and Surgery, University of University of Milan-Bicocca, Milan, Italy.
\end{abstract}

\title{
Acknowledgements
}

Supported by Associazione Nazionale di Lotta all'AIDS (ANLAIDS) Sezione Lombarda (Italy) and Società Italiana di Terapia Antimicrobica (SITA).

\section{Compliance with ethical standards}

\section{Conflicts of interest}

On behalf of all authors, the corresponding author states that there is no conflict of interest.

Accepted: 26 December 2018

Published online: 22 January 2019

\section{References}

1. Ruppé E, Lisboa T, Barbier F (2018) The gut microbiota of critically ill patients: firs steps in unexplored word. Intensive Care Med. https://doi. org/10.1007/s00134-018-5309-3

2. Freedberg DE, Zhou MJ, Cohen ME, Annavajhala MK, Khan S, Moscoso DI, Brooks C, Whittier S, Chong DH, Uhlemann AC, Abrams JA (2018) Pathogen colonization of the gastrointestinal microbiome at intensive care unit admission and risk for subsequent death or infection. Intensive Care Med. https://doi.org/10.1007/s00134-018-5268-8

3. Wischmeyer P, McDonald D, Knight R (2016) Role of the microbiome, probiotics and dysbiosis therapy in critical illness. Curr Opin Crit Care. https:// doi.org/10.1097/mcc.0000000000000321

4. Lu W, Yu J, Ai Q, Liu D, Song C, Li L (2014) Increased constituent ratios of Klebsiella sp., Acinetobacter sp., and Streptococcus sp. and a decrease in microflora diversity may be indicators of ventilator-associated pneumonia: a prospective study in the respiratory tracts of neonates. PLOS ONE 9(2):e87504. https://doi.org/10.1371/journal.pone.0087504

5. Zakharkina T, Martin-Loeches I, Matamoros S, Povoa P, Torres A, Kastelijn JB, Hofstra JJ, de Wever B, de Jong M, Schultz MJ, Sterk PJ, Artigas A, Bos LDJ (2017) The dynamics of the pulmonary microbiome duringmechanical ventilation in the intensive care unit and the association with occurrence of pneumonia. Thorax 72:803-810. https://doi.org/10.1136/thora xjnl-2016-209158 